\title{
The Study on the Modularity Design Principles of the Packaging and the Styling of Yacht
}

\author{
Gangqiang Zheng, Yuwei Deng, Jingting Du* \\ School of Art and Design, Wuhan University of Technology, Wuhan, China \\ Email: zhenggq@whut.edu.cn,943307698@whut.edu.cn, 402386172@qq.com
}

Received 5 August 2016; accepted 20 August 2016; published 23 August 2016

Copyright (C) 2016 by authors and Scientific Research Publishing Inc.

This work is licensed under the Creative Commons Attribution International License (CC BY). http://creativecommons.org/licenses/by/4.0/

\section{(c) (i) Open Access}

\begin{abstract}
In the 21th Century, yachts gradually enter the Chinese market. For the purpose of promoting the innovation of yaht design, the article discussed about the modularity design principle of the packaging and styling of yacht and analyzed the confluenced system and the design considerations of the modularity design of the yacht. Through the analysis of the the physical structure of the yacht, the design theory and the design application, the article explains the confluenced and complementary principle of design. The priniples combine together, making the the modularity design systems of the packaging and the styling of yacht more perfect. It laid the foundation for the design innovation of the yacht.
\end{abstract}

\section{Keywords}

Yacht, Packaging, Styling, Modularity, Design

\section{Introduction}

Yacht design in China develops quickly these years. The study of yacht modularity design is in need. The article emphatically analyzes the interdependence principle and the correspondence principle of yacht packaging system. It laid the foundation for the design innovation of the yacht.

Yacht is a kind of high-end water transportation which has many functions such as relaxation, social contact and exercise. Since the 21 st century, with the progress of science and technology, the number of the large-scale and intelligent yacht increases, which makes the complexity of the system integration constantly increase (Wuhan University of Technology Ships \& Yachts design research team, 2011). To get the method and the technol-

${ }^{*}$ Corresponding author. 
ogy of yacht design, we must analyze the modularity system of the packaging and the styling of yacht. Through identifying the inherent law and the design principle, the design innovation of yacht can be promoted.

On the one hand, the "hard" design should be considered in the modularity design of packaging and styling of yacht such as the planning and layout of the area and the packaging and interface design for cabin, making it reach the corresponding technical standards (Ren, Liu, \& Shi, 2009). On the other hand, "soft" design should also be taken into consideration. We should follow the principle of "People oriented, goods serve people" to meet the human's requirements of physical characteristics, living habits and the regularity of human activities. Also, we should deeply combine the yacht cabin design and the yacht styling together to make the styling and the packaging become a whole and make the yacht cabin full of life interest and cultural connotations. All of these help us eventually reach the design goals of getting reasonable packaging layout, up-to-standard cabin size, elegant and appropriate colors, suitable packaging style and the reasonable separation of the fire prevention structure.

The modularity design of the packaging and the styling of yacht should both follow the ship design principles and the general principles of product design which includes practicability, aesthetics and economics (Hou, 2003). The multiple design categories such as the yacht cabin packaging design, the styling design, the hull design, the structure design and the mechanical design compose the overall design system of yacht. Each component design is just one part of the overall design. The modularity design of the yacht cabin packaging should be based on human needs. Through the demand analysis of market research and user research, it explores the phenomena together with the laws of the interdependence of yacht packaging system and the styling. It also studies the blending characteristics and principles which include the multidisciplinary cross and communion and the blending of the true and false and the art and science of the styling and packaging (the latter one contains the blending of rational design, production and manufacturing, and the cultural connotation and social value). The above laid the foundation of styling innovation, function innovation and structure innovation of yacht.

\section{The Interdependence Principle of the Yacht Packaging System}

The yacht cabin packaging and the external styling are interdependent. They have an indivisible dualistic unification relation. The interdependence design principle of the yacht styling and the cabin packaging can be analyzed from these parts.

As a general view, the yacht modularity design includes the yacht styling design and the cabin packaging environment design which equals to the "real" styling and the "virtual" packaging. In the meantime, there are also two aspects such as "the styling in the packaging" and "the packaging in the styling" included. In this theory, "styling" refers to the overall visual styling of the yacht external. It also refers to the styling of the facilities and the equipment in the internal and the external of the cabin. "Packaging" contains three meanings: 1) the macro environment packaging of yacht; 2) the outer packaging which related to the yacht function; 3 ) the internal yacht cabin packaging which meets the requirements of the main function.

The "dependency" refers to the dialectical relationship such as the interdependency and the mutually cause and effect of the external yacht styling and the internal cabin packaging. The interdependency of the yacht styling and the cabin packaging makes it different from other productions. Using this as a starting point, we can explore the essence of the yacht design. The characteristics and the mechanism is embodied in the following two points: 1) It is the overall external "entity" of the yacht enclosing that makes up the internal "virtual" yacht cabin packaging which meets the requirements of sojourn, relaxation and manipulation. In other words, the 'real' styling makes up the "virtual" packaging which is similar with that the walls of containers make up the functional space of containers; 2) The changes of the function packaging can directly influence the external styling. For example, the yacht "flybridge", the expanding leisure area and the side face of the flap are all classic cases of that function packaging rebuilds the yacht styling.

The dependency between yacht styling and cabin packaging can be seen in Picture 1 . The left one shows the overall modeling of a 39 feet sport yacht while the right one shows the modeling of a 148 feet liner. From those two designs, we can figure out that there is a character of dependence and indivisibility between yacht styling and cabin packaging. From which we can also find that the design of yacht should also follow the basic law of "the integrated design of packaging and styling". Through summarizing and analyzing the phenomenon of interdependency between yacht styling and packaging, we can find that "the integrated design of packaging and styling" is one of the basic laws. Combining "real" and "virtuality" to finish the design of yacht can help to create new styling and packaging (as it showed in [1], Picture 1). 


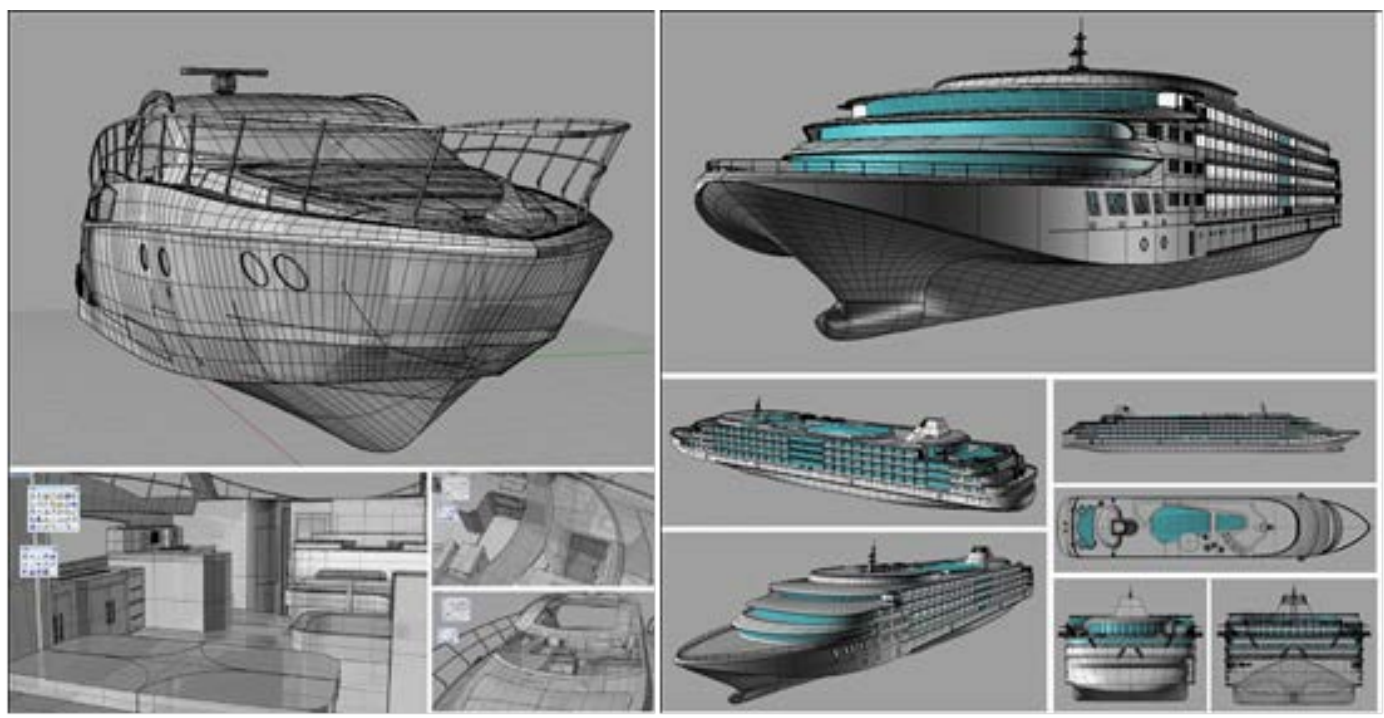

Picture 1. The interdependency between the yacht and cruise modelling and the cabin packaging (the modelers: Wan Li, Song Wei).

\section{The Correspondence Principle of the Yacht Packaging System}

"Blending" means cross, integration, complementarity and coincidence. On the foundation of understanding the interdependency of yacht packaging system, we can start our mechanism study of the causes and the phenomena which generated by the blending of the yacht styling and cabin packaging. We can also analyze the coupling correspondences between the phenomenon and the causes of the complementarity, explore the relation, the characteristics and the laws of coexistence, start the laws study of the related characteristic of yacht styling and cabin packaging and explore the advanced and effective design patterns to make effects on the optimal design and grade enhancing of yacht in practical applications.

\section{The analysis of the blending system of yacht modularity design}

1) About the local modularity design system of yacht: The most effective way to study and research on the local yacht design is from imitation, absorption, integrated innovation to local recreation. The integration of the combination of yacht styling and cabin packaging design main includes the overall design of yacht styling (the exterior styling elements include integral style, the superstructure styling and layout and the color design of the exterior hull), the yacht cabin packaging design (the interior environment elements include the rational layout of the interior cabin packaging, the creative design of the visual environment and the layout of the interior furnishings design) and the study of the blending characteristics of the inter-coordination between yacht styling and cabin packaging design (Dennis, Wixom, \& Roth, 2010). The purport is to correctly grasping the internal relations and differences between the advanced design elements and local culture system. Besides, it aims to locally translate, understand and integrate the good design concept to cater to market demands to finally reach the state that all design elements blend together.

2) About the modularity design elements of yacht blending characteristics: To analyze and integrate the elements which should be considered when designing according to the normal yacht design process, we can ascertain the close connection between yacht design and the following elements such as design aesthetics, the ship engineering design and the system design. The design art holds an important position in blending system of yacht design. In the practical yacht design, the design art and the aesthetics play an important role in connecting the user's function requirements and ship engineering technology and become an essential shared interface of their unification. In the meantime, the expanding of the function demands and the introduction of the new energy, new structure, new technology and new materials provide the continual creation inspiration for yacht design.

The yacht design aesthetics is the key element of yacht modularity design. The design elements include shape, light and color. Shape equals to the exterior styling. It is the foundation of the design mood. The size, the position, the linetype and the angle of shape are all important design elements of design style. The excellent yachts 
usually attract the world's attention by their special design aesthetics conception. For example, the sharp hard lines of Wally, the mellow graceful surfaces of Princess and the staggered angle intersections of Sunseeker are all new individual elements which are produced basing on the design aesthetics (Wuhan University of Technology Ships \& Yachts design research team, 2011).

The various properties of the yacht fundamentally depend on the related parameters in the ship engineering design. So in the yacht design, the ship engineering design plays an important role. It is the fundamental element of yacht design. Particularly, the underwater part analysis about the flow resistance and the layout of hull porthole and engineering mechanics design are all important indexes of improving the motility, safety and energy saving of yacht itself. As a result, the proper analysis and design of the ship engineering become a significant element of yacht design (Dennis, Wixom, \& Roth, 2010).

Yacht is a complicated industrial product which combines multiple areas. The system design is a methodology which blends and integrates the various design elements. The modularity design conception decomposes the various design elements in yacht design and simplifies, classifies and integrates them to get a huge database of yacht design elements. It lays the foundation of future design and mass production.

\section{The combination of the blending and complementary principles of modularity system}

1) About the foundation of blending design principles of modularity systems: "Blending" means integration and interaction. It refers to the contact, the mixture and the integration of different elements. The blending in yacht design can be interpreted in several aspects such as physical structure, design theory and design application.

The first aspect refers to the yacht physical structure. The confluence and complementation principle reflects in the blending between styling and packaging especially in flybridge area, the cockpit area and the swimming area. The styling and the packaging blend together, forming the special characteristic of yacht.

The second aspect refers to the design theory. The confluence and complementation principle widely existed in the process of various design systems integration. Because of the complexity of yacht design, using existed design science system to analyze and explain the yacht design is an effective way to quickly understand the yacht design concept system. From this aspect, integrating and blending the existed science theory is an important fundamental theory of yacht design.

The third aspect refers to the design application. Compared to the significance of the first aspect, this one is more closed to the actual production. The confluence and complementation theory contains the whole process from yacht design to concrete realization. The differences and relations of design demands between yacht hull styling and cabin packaging are destined to the contradictions and common features between them. Integrating the relations and the contradictions between styling and cabin packaging is an important part of yacht design practice. How to cope with the contradictions between them is also the key to reflect the level of the designers (Feng, Liu, \& Chang, 2009).

2) The analysis of the influence of the confluence and complementation modularity design principle: The confluence and complementation principle is not only the systematic integration to the yacht design system but also the compromise and the blending to the concrete design objectives. In the process of yacht design, there are a lot of unavoidable contradictions such as contradictions between exterior and use and cost and performance. Among them, the foremost contradiction is the contradiction between exterior and use which means the contradictions between styling and cabin packaging. The yacht styling and cabin packaging can be divided into three parts: the yacht products, the superstructure and the hull and the all parts of yacht (the hull, the stem, the flybridge, the spatial association and interior packaging). Now we'll analyze the influence of the confluence and complementation design principle in different parts to styling and cabin packaging.

The hull which includes the main hull, the porthole and the skylight (includes some facilities like platform trap door) is an important part of the yacht exterior. It is also the main part of making up the yacht side facade structure. This part laid a line tone of yacht. It is where the verve of yacht reflects. In the opposite window position to the side facade, the solar orientation problem of its interior packaging will be considered. The corresponding windows will be set up in the master bedrooms and guest bedrooms. The size and the shape of the windows would be controlled according to the room size. In the after body of the hull, the cabin is usually totally-enclosed. In this way, we can not only coordinate the position and the layout of the side façade windows but also ensure the firm of the hull and reinforce the engine section which is the dynamic core of yacht. It is the perfect combination of the exterior and safety. In the fore-body of the hull, as the downsizing of the cabin packaging and the limitation of the function area, it usually tends to be a small-sized leisure area and reserves a chain 
well for the yacht. Making a more effective use of cabin packaging combining styling and giving consideration to both exterior and safety and practicability are the concrete reflection of the confluence and complementation principle.

The porthole contains the upper deck and the main deck. The concrete structure includes stem, weigh anchor, board and corridor. The concrete structure which matches functions can also include leisure sunbathing area, crane and boat storage area. The porthole is the main part of forming the frontage of yacht. It is also the "face" of the yacht. The structure of the porthole directly determines the first impression of the yacht to people so that many international-brand yachts have their own house style porthole structure. The structure of the sunbathing area and the windshield is the structure that embodies the yacht design style most. As an important leisure area, the sunbathing area has a half open structure which have the characteristics of both interior packaging and exterior styling. Entering the sunbathing area is equal to entering an outdoor half open area. The layout of the sofa and the deck chair, and the set of the tables are all designed based on the interior packaging structure. Seeing from the exterior, the sunbathing area is not only an interior packaging structure. It is also influenced by the overall exterior design thought and plays a role in exterior design style. So the porthole is an important reflection of the confluence and complementation design. It is the meeting-point of those two design principles (as is showed in Picture 2 left [1]).

The stern includes stairs, pillar fences, cabin, cockpit and swimming area. According to some concrete functions, it main also includes springboard, boat parking area and crane. Among them, the cockpit includes possible bars and restaurants. Similar to the sunbathing area in porthole, it is a half open area. It is an important parts of yacht leisure area.

The flybridge deck is the concentrated embodiment of the blending characteristics of yacht styling and cabin. It takes a considerable position in the overall structure of yacht. The flybridge deck can be divided into two parts: driver zone and leisure area. It can also include the boat storage area and crane. The driver zone is an important part of man-machine interaction. Its design should combine the interior packaging design and ergonomic theory. In the meantime, the characteristic of the yacht overall styling should be considered too. The special structure and function makes it the central feature of flybridge deck. A good design of bridge can usually make the finishing point. The leisure area includes sofa, stairs and surfing pools. The layout of sofa and table, the decorative pattern of floor, the front of the stairs are all important parts that make up the flybridge deck structure design. And even the tiny changes can reflect different styles. For the yacht for business and leisure, how to distribute the basic elements of flybridge deck to find an important balance, which is the hallmark of design, is the aspect that designers should most pay attention to. The flybridge includes ceiling of flybridge deck, radar mount, communications equipment, crane, windshield and stainless steel handrails. Like the top of the building, the design of flybridge is a remarkable characteristic of the yacht that sails on the sea. The structure of the flybridge leads to the characteristic of combination of interior packaging and styling and condemns it to an important reflection of the blending design principle in yacht design.

As a part of interior packaging, the styling structure of yacht should be taken into more consideration which includes public packaging (salon area, dining area, bars and living rooms), private packaging (master bedrooms, VIP bedrooms, crew packaging and bathrooms) and work packaging (driver zone, cab, kitchen and cabin) (Ren, Liu, \& Shi, 2009). The layout of different function area combining the yacht exterior styling design, properly
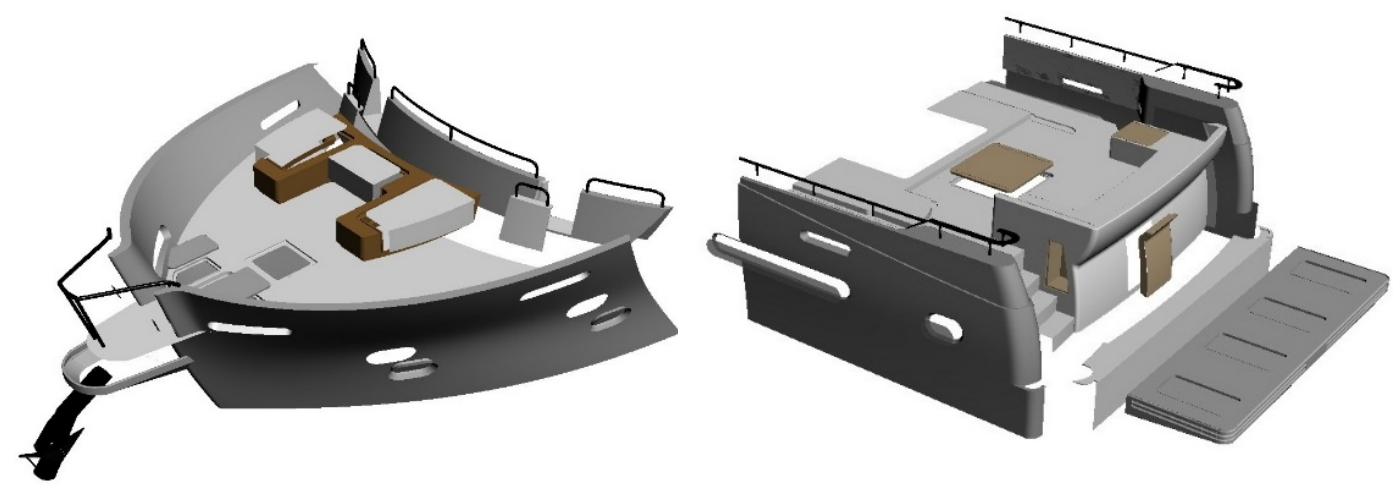

Picture 2. The separation of the packaging modules of yacht porthole and stern (the modelers: Gao Xi). 
using the available packaging which is scarce in interior packaging to accurately handle the packaging and styling design is a kind of blending in the principle aspect.

\section{Conclusion}

The article discusses about the principle of yacht cabin packaging modularity design, expounding the interdependence principle and the confluence and complementary principle of yacht packaging. According to the article, the interdependence principle is embodied in that the yacht cabin packaging and the external styling are interdependent. They have an indivisible dualistic unification relation. The article analyzes the confluenced system and the design considerations of the modularity design of the yacht. Through the analysis of the physical structure of the yacht, the design theory and the design application, the article explains the confluenced and complementary principle of design. It laid the foundation for the design innovation of the yacht.

\section{Funding}

This project was subsidized by the National Social Science Fund art program (program number: 13CB116); China Scholarship Council (201406955046); Teaching and Research Program, Hubei province (program number: 2012123).

\section{References}

Wuhan University of Technology Ships \& Yachts design research team. (2011). The Repository and Database of Ship Design. Wuhan: Wuhan University of Technology.

Ren, N., Liu, J. Y., \& Shi, H. B. (2009). The Study on the Breakdown Structure of Ship Engineering. Shipbuilding Technology, 3, 112.

Hou, L. (2003). The Study on the Modularity Developing Theory, Method and Application of Mechanical Product. PhD Thesis, Hangzhou: Zhejiang University.

Dennis, A., Wixom, B. H., \& Roth, R. M. (2010). The System Analysis and Design (pp. 4-15). Beijing: Posts \& Telecom Press.

Feng, B. W., Liu, Z. Y., \& Chang, H. C. (2009). The Appliance of Multi-Interdisciplinary Optimal Design in Boat Elementary Design. China Shipbuilding, 50, 109-116. (In Chinese)

\section{Submit or recommend next manuscript to SCIRP and we will provide best service for you:}

Accepting pre-submission inquiries through Email, Facebook, LinkedIn, Twitter, etc.

A wide selection of journals (inclusive of 9 subjects, more than 200 journals)

Providing 24-hour high-quality service

User-friendly online submission system

Fair and swift peer-review system

Efficient typesetting and proofreading procedure

Display of the result of downloads and visits, as well as the number of cited articles

Maximum dissemination of your research work

Submit your manuscript at: http://papersubmission.scirp.org/ 